CARDIOVASCULAR MEDICINE

\title{
Rapid access arrhythmia clinic for the diagnosis and management of new arrhythmias presenting in the community: a prospective, descriptive study
}

\author{
J L Martins, K F Fox, D A Wood, D C Lefroy, T J Collier, N S Peters
}

Heart 2004;90:877-881. doi: 10.1136/hrt.2003.021493

See end of article for authors' affiliations

.....................

Correspondence to:

Dr K F Fox, Cardiovascular Medicine, National Heart and Lung Division, Charing Cross Campus, Imperial College, London W6 8RF, UK: k.fox@imperial.ac.uk

Accepted 27 October 2003
Objective: To investigate whether a rapid access approach is useful for the evaluation of patients with symptoms suggestive of a new cardiac arrhythmia.

Design: Prospective, descriptive study.

Setting: Secondary care based rapid access arrhythmia clinic in West London, UK.

Participants: Patients referred by their general practitioner or the emergency department with symptoms suggestive of a new cardiac arrhythmia.

Main outcome measures: Number of patients with a newly diagnosed significant arrhythmia. Number of patients with diagnosed atrial fibrillation. Number of eligible, moderate, and high risk patients treated with warfarin.

Results: Over a 25 month period 984 referrals were assessed. The mean age was 55 years (range 20-90 years) and $56 \%$ were women. The median time from referral to assessment was one day. A significant cardiac arrhythmia was newly diagnosed in $40 \%$ of patients referred to the RAAC. The most common arrhythmia was atrial fibrillation, with 203 new cases (21\%). Of these, 74\% of eligible patients over 65 were treated with warfarin. Other arrhythmias diagnosed were supraventricular tachycardias (127 $(13 \%))$, conduction disorders $(43(4 \%))$, and non-sustained ventricular tachycardia (21 (2\%)). Vasovagal syncope was diagnosed for 53 patients $(5 \%)$. The most frequent diagnosis was symptomatic ventricular and supraventricular extrasystoles (355 (36\%)).

Conclusion: A rapid access arrhythmia clinic is an innovative approach to the diagnosis and management of new cardiac arrhythmias in the community. It provides a rapid diagnosis, stratifies risk, and leads to prompt initiation of effective treatment for this population.
$\mathrm{T}$ he diagnosis of cardiac arrhythmias can be difficult in primary care and the waiting time for a first appointment with a consultant cardiologist may be several months. The National Service Framework for coronary heart disease created a momentum for change in cardiological practice in the UK, with the expansion of rapid access chest pain clinics as a favoured service model. ${ }^{1}$ We have previously reported experience in the establishment of rapid access clinics for chest pain and heart failure and proposed that this service could be extended to arrhythmias. ${ }^{23}$

Palpitations, presyncope, and syncope are common symptoms that may be linked to serious tachyarrhythmias or bradycardias. Atrial fibrillation is the most common arrhythmia in the population and can lead to a threefold increased risk of developing heart failure and a fivefold increased risk of stroke. People with atrial fibrillation have a mortality rate nearly double that of age and sex matched controls. ${ }^{4-6}$ Despite abundant clinical evidence that warfarin is an effective treatment for the prophylaxis of thromboembolism in these patients, ${ }^{7-12}$ oral anticoagulation remains suboptimal in both primary and secondary care, with treatment rates of 21$50 \% .^{13-17}$

We established a rapid access arrhythmias clinic (RAAC) in the cardiology department of Charing Cross Hospital in West London. The aims of the RAAC were, firstly, to provide a rapid diagnosis of significant cardiac arrhythmias in patients presenting for the first time in the community; secondly, to describe the clinical characteristics of incident cases of atrial fibrillation; and thirdly, to facilitate the early initiation of warfarin and other treatments for atrial fibrillation based on European Society of Cardiology guidelines. ${ }^{18}$
This paper describes the organisation of the RAAC and the characteristics of the patients seen, and discusses the role of a rapid access hospital based strategy for identifying and managing incident (new) arrhythmias in the community.

\section{METHODS}

\section{Population}

Charing Cross Hospital is a large undergraduate teaching hospital with secondary cardiology services based in West London. It serves a population of between 150 000-200 000 that is both ethnically and socioeconomically diverse.

\section{Rapid access arrhythmia clinic}

All patients presenting to their general practitioner or to the emergency department with a first presentation of symptoms or signs suggestive of a cardiac arrhythmia were eligible for referral to this new service. Before the opening of the clinic, all general practitioners within our catchment area were sent letters advertising this new service, building upon the already established rapid access chest pain clinic at this hospital. The service was also advertised during the induction programme of new senior house officers in the emergency department.

The clinic was open each weekday between $9.30 \mathrm{am}$ and $12.30 \mathrm{pm}$ and no appointments were needed. All patients had an ECG followed by a clinical assessment by the doctor running the clinic (JLM). Those with a history of palpitations, presyncope, or syncope had a 24 hour Marquette Hellige Holter monitor system fitted the same day. All patients with confirmed atrial fibrillation also underwent chest radiography and a transthoracic echocardiography, and were individually risk stratified as low, intermediate, or high 


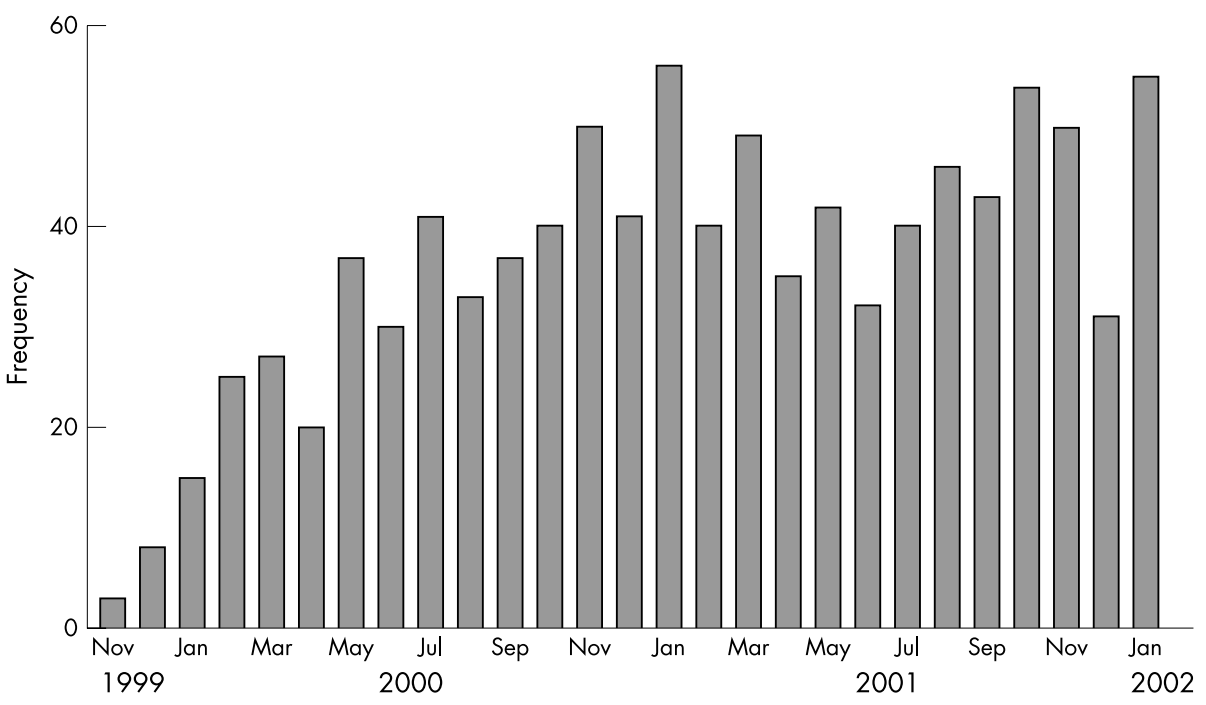

Figure 1 Number of patients seen each month.

risk for thromboembolism according to European Society of Cardiology guidelines. ${ }^{18}$ Low risk patients were aged less than 65 years without any high risk features (previous thromboembolism, heart failure, hypertension, diabetes mellitus, left ventricular dysfunction, coronary heart disease) and were treated with aspirin or no antithrombotic. Warfarin was started for patients assessed as moderate to high risk for stroke (age greater than 65 years or less than 65 years with high risk features) provided there were no clinical contraindications.

In cases where there was diagnostic difficulty further investigations such as event (loop) recording or tilt testing were arranged. The initial diagnosis was based on the clinical assessment and ECG. The final diagnosis was made after reviewing the results of all further investigations. Patients with a cardiac arrhythmia that required hospital follow up were reviewed as cardiology outpatients or referred to a dedicated arrhythmia clinic led by a consultant electrophysiologist (DCL). All data were entered on to a Microsoft Access (Microsoft Corp, Redmond, Washington, USA) database designed for the clinic. A full report detailing the diagnosis, results of investigation, and proposed management was automatically generated from the database and sent to the general practitioner, usually within 24-48 hours of assessment.

\section{Definitions}

"Atrial fibrillation" includes patients with atrial flutter, occurring either with atrial fibrillation or alone. "Supraventricular tachycardia" includes focal atrial tachycardia, atrioventricular nodal re-entrant tachycardia, and atrioventricular re-entrant tachycardia. "Conduction disease" includes sinoatrial disease and all forms of heart block. "Non-sustained ventricular tachycardia" is defined as six or more ventricular complexes. "Possible arrhythmia" is a clinical diagnosis based on symptoms of abrupt onset and offset but without ECG documentation of arrhythmia.

\section{RESULTS}

\section{Clinic operation and characteristics of patients assessed}

The following results describe a 25 month period from 23 November 1999 to 31 January 2002. During this time 998 patients were referred to the RAAC but 13 were excluded because of previously diagnosed arrhythmias and two because they were referred from another hospital. This report is based on the 984 (99\%) appropriate referrals.
Referral patterns stabilised after five months and a background seasonal fluctuation was observed (fig 1). The referral rate was subsequently $1.0-1.3 / 100000$ population each weekday. The mean number of patients seen each month was 42 (range 30-56). The number of patients seen each weekday varied from none to seven, with a mean of two (fig 2). The median time from referral to assessment was one day (interquartile range $0-6$ ).

Table 1 shows the baseline characteristics. The mean age was 55 years (range $20-90$ years) and $56 \%$ were women. The majority of referrals were from local general practitioners $(81 \%)$, with an additional $15 \%$ of referrals from the emergency department. The most prevalent medical history was hypertension (26\%) followed by anxiety or depression $(13 \%) ; 24 \%$ of patients were current smokers and $19 \%$ admitted to excessive alcohol consumption. The most common presenting symptom was palpitations (76\%), followed by dyspnoea, dizziness, chest pain, and syncope (table 2).

\section{Investigations, management, and diagnosis}

As part of their initial assessment all patients had an ECG, 97\% had blood tests, and 93\% were fitted with a Holter monitor according to protocol (table 3 ). In addition, those with atrial fibrillation underwent echocardiography (95\%) and chest radiography (93\%). A further $14 \%$ and $12 \%$ of patients without atrial fibrillation underwent echocardiography and an exercise tolerance test, respectively, because of

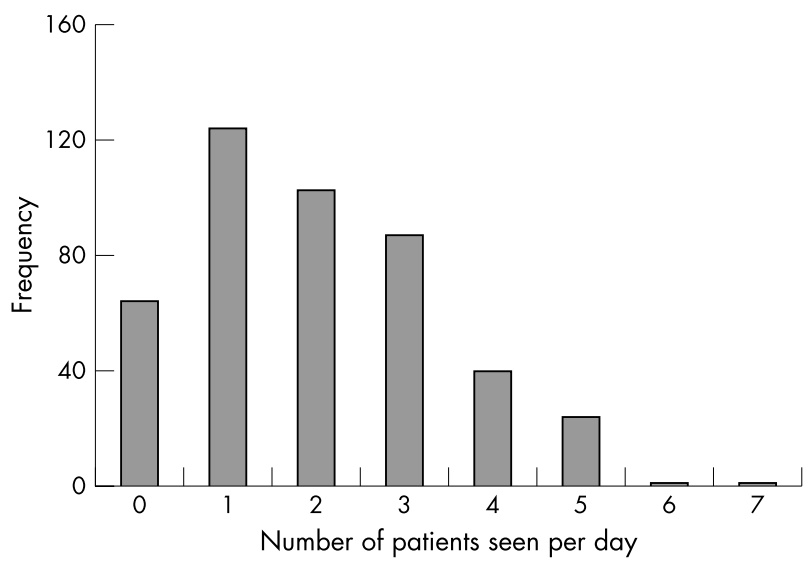

Figure 2 Number of patients seen each weekday. 
Table 1 Baseline characteristics of 984 patients referred to the rapid access arrhythmia clinic

\begin{tabular}{ll}
\hline Category & Number (\%) \\
\hline Age (years) & \\
$<40$ & $268(27.2 \%)$ \\
$40-59$ & $303(30.8 \%)$ \\
$60-79$ & $316(32.1 \%)$ \\
$\geqslant 80$ & $97(9.9 \%)$ \\
Mean (SD) & $55(19.0 \%)$ \\
Sex & \\
Male & $429(43.6 \%)$ \\
Female & $555(56.4 \%)$ \\
Source & $792(80.5 \%)$ \\
General practitioner & $140(14.2 \%)$ \\
Emergency department & $52(5.3 \%)$ \\
Other & $801(81.4 \%)$ \\
Ethnic group & $183(18.6 \%)$ \\
White & \\
Non-white & $456(46.3 \%)$ \\
Smoking & $295(30.0 \%)$ \\
Never smoker & $233(23.7 \%)$ \\
Former smoker & \\
Current smoker & $115(27.0 \%)$ \\
Excess alcohol* & $71(13.0 \%)$ \\
Men & $186(19.0 \%)$ \\
Women & $255(26 \%)$ \\
Overall & $129(13 \%)$ \\
Medical history & $97(10 \%)$ \\
Hypertension & $52(5 \%)$ \\
Anxiety/depression & $45(5 \%)$ \\
Lung disease & $42(4 \%)$ \\
Coronary artery disease & $27(3 \%)$ \\
Other vascular disease & \\
Diabetes & \\
Other cardiac disease & \\
\hline & \\
\hline &
\end{tabular}

*Defined as consuming $>21$ units a week (men) and $>14$ units a week (women).

clinical indications, mostly to evaluate murmurs or chest pain. Only $5 \%$ of patients had an event recorder and $2 \%$ were referred for tilt testing.

Two thirds of patients were discharged back to their general practitioners after the first visit to the RAAC and a third were followed up as cardiology outpatients or referred to the arrhythmia clinic. Twenty two patients $(2 \%)$ were admitted to hospital because of decompensated heart failure (13), severe anaemia (2), acute myocardial infarction (1), or symptomatic high grade atrioventricular block (1). Five were admitted for immediate cardioversion of atrial fibrillation.

Table 4 shows the results of the initial and final diagnoses. The most frequent diagnosis recorded for 355 (36\%) patients was symptomatic ventricular and supraventricular extrasystoles; $54 \%$ had greater than 10 extrasystoles an hour (or $>240$ in 24 hours) and $39 \%$ had greater than 1000 extrasystoles a day. The most common significant arrhythmia was atrial fibrillation $(21 \%)$. Thirty one per cent of patients

\begin{tabular}{ll} 
Table 2 Presenting symptoms & \\
\hline Symptom & Number (\%) \\
\hline Palpitations & $745(75.7 \%)$ \\
Dyspnoea & $311(31.6 \%)$ \\
Dizziness & $307(31.2 \%)$ \\
Chest pain & $253(25.7 \%)$ \\
Syncope & $91(9.2 \%)$ \\
Asymptomatic/coincidental & $71(7.2 \%)$ \\
Leg swelling & $39(4.0 \%)$ \\
Paroxysmal nocturnal dyspnoea & $36(3.7 \%)$ \\
Orthopnoea & $23(2.3 \%)$ \\
Symptom of embolic event & $12(1.2 \%)$ \\
Diuresis & $8(0.8 \%)$ \\
\hline
\end{tabular}

Table 3 Investigation and management

\begin{tabular}{ll}
\hline Investigations & Number (\%) \\
\hline ECG & $984(100 \%)$ \\
Blood tests & $955(97 \%)$ \\
Holter monitoring & $912(93 \%)$ \\
Echocardiography & $311(32 \%)$ \\
Chest radiography & $187(19 \%)$ \\
Exercise stress test & $119(12 \%)$ \\
Event recorder & $52(5 \%)$ \\
Tilt test & $16(2 \%)$ \\
Further management & \\
Discharged to general practitioner & $659(67 \%)$ \\
Followed up in conventional cardiology clinic & $242(25 \%)$ \\
$\quad$ Referred to specialist arrhythmia clinic & $83(8 \%)$ \\
Admitted to hospital & $22(2 \%)$ \\
Referred for pacemaker & $21(2 \%)$ \\
Referred for coronary angiography & $9(1 \%)$ \\
\hline
\end{tabular}

Table 4 Diagnoses for 984 patients assessed in the rapid access arrhythmia clinic

\begin{tabular}{lll}
\hline Assessment & Initial number (\%) & Final number (\%) \\
\hline Extrasystoles & $337(34.3 \%)$ & $355(36.1 \%)$ \\
Atrial fibrillation & $173(17.6 \%)$ & $203(20.6 \%)$ \\
Anxiety/stress & $181(18.4 \%)$ & $177(18.0 \%)$ \\
Other SVTs & $33(3.4 \%)$ & $127(12.9 \%)$ \\
Syncope & $53(5.4 \%)$ & $53(5.4 \%)$ \\
Conduction disease & $23(2.3 \%)$ & $43(4.4 \%)$ \\
Possible arrhythmia & $163(16.6 \%)$ & $40(4.1 \%)$ \\
Non-sustained VT & $1(0.1 \%)$ & $21(2.1 \%)$ \\
Other & $284(28.9 \%)$ & $308(31.4 \%)$ \\
\hline
\end{tabular}

Some patients have more than one diagnosis.

SVT, supraventricular tachycardia; VT, ventricular tachycardia.

had other diagnoses, either as their primary diagnosis (7\%) or as a secondary diagnosis (24\%), including $78(8 \%)$ with thyroid disease, anaemia, diabetes, coronary artery disease, and heart failure.

\section{Characteristics and antithrombotic treatment of patients with atrial fibrillation}

Table 5 shows the clinical characteristics of the 203 patients with atrial fibrillation. The mean age was 74 years and the majority $(71 \%)$ were $\geqslant 65$ years of age at first presentation. In contrast to patients without atrial fibrillation, among those with atrial fibrillation, the most common presenting symptom was dyspnoea, followed by palpitations, chest pain, and dizziness. The overall treatment rate with warfarin of eligible patients over 65 with atrial fibrillation was $74 \%$. Contraindications in this age group were present among $16 \%$ of men and $21 \%$ of women. Among all ages warfarin was initiated for a total of $111(55 \%)$ patients; aspirin was given to $80(39 \%)$ patients, and $12(6 \%)$ patients did not receive any antithrombotic agent.

\section{DISCUSSION}

Palpitations and syncope are common symptoms, which may be caused by serious, yet treatable arrhythmias. Effectively and promptly distinguishing the serious from the benign and helping all groups of patients effectively are the challenges to those developing innovative models of care. We proposed that the rapid access model would be applicable to patients presenting in primary care with suspected cardiac arrhythmias. We established a patient centred service modelled closely on our rapid access chest pain and heart failure clinics. Given the periodic nature of paroxysmal arrhythmias, 
Table 5 Characteristics of 203 patients with new atrial fibrillation

\begin{tabular}{ll}
\hline Category & Number (\%) \\
\hline Male & $110(54 \%)$ \\
Age (years) & $13(6 \%)$ \\
$<40$ & $31(15 \%)$ \\
$40-59$ & $107(53 \%)$ \\
$60-79$ & $52(26 \%)$ \\
$\geqslant 80$ & $74(36.5 \%)$ \\
Medical history & $27(13.3 \%)$ \\
Hypertension & $13(6.4 \%)$ \\
Coronary artery disease & $6(3.0 \%)$ \\
Previous stroke & $8(3.9 \%)$ \\
Rheumatic fever & $1(0.5 \%)$ \\
Diabetes & $32(16 \%)$ \\
Heart failure & $114(67 \%)$ \\
Presenting symptoms & $96(56 \%)$ \\
Asymptomatic & $59(35 \%)$ \\
Dyspnoea & $58(34 \%)$ \\
Palpitations & $12(7 \%)$ \\
Chest pain & $7(4 \%)$ \\
Dizziness & $5(3 \%)$ \\
Syncope & $121(60 \%)$ \\
Embolic event & $82(40 \%)$ \\
Diuresis & $164(81 \%)$ \\
Arrhythmia subtypes & $25(12 \%)$ \\
Sustained & $14(7 \%)$ \\
Paroxysmal & $37(93 \%)$ \\
AF alone & $7(39 \%)$ \\
AF and atrial flutter & $31(97 \%)$ \\
Atrial flutter alone & $11(41 \%)$ \\
Anticoagulation of patients over 65 years without contraindications \\
Men \\
Sustained AF $(n=40)$ \\
Paroxysmal AF ( $n=18)$ & \\
Women & \\
Sustained AF $(n=32)$ & \\
Paroxysmal AF $(n=27)$ & \\
\hline AF, atrial fibrillation. & \\
& \\
\hline &
\end{tabular}

it seems intuitive that prompt assessment when patients are most symptomatic would increase the chances of a definitive diagnosis compared with conventional outpatients. In the RAAC, the median wait from referral to consultation was only one day. New arrhythmias were referred to a consultant electrophysiologist and patients with a new diagnosis of atrial fibrillation were fully evaluated in one consultation. This resulted in early risk stratification and initiation of warfarin, which may improve their prognosis, as the risk of stroke is reported to be highest in the early months after diagnosis of atrial fibrillation. ${ }^{19} 20$

The RAAC model also has value for patients without serious disease. Patients who experience palpitations are frequently anxious because they believe they have some serious cardiac pathology. A long wait for specialist investigations is likely to increase anxiety and disability, as has been shown among patients waiting for coronary artery surgery, ${ }^{21}$ and makes it more difficult to reassure patients with benign symptoms.

The initial clinical assessment based on careful history taking, physical examination, and resting 12 lead ECG proved to be very accurate in assessing patients with symptomatic extrasystoles and anxiety related symptoms (table 4 ). Holter monitoring made little difference to their final diagnosis, suggesting that this is not routinely required when the initial assessment points to one of these diagnoses. It proved most useful in recording other supraventricular tachycardias (an additional 94 of 127 cases), non-sustained ventricular tachycardia (an additional 20 of 21 cases), conduction disease (an additional 20 of 43 cases), and to a lesser extent atrial fibrillation (an additional 33 of 203 cases), establishing a diagnosis for 123 patients with an initial assessment of "possible arrhythmia". This experience concurs with the advice of Zimetbaum and Josephson ${ }^{22}$ in their comprehensive review of the evaluation of patients with palpitationsnamely, that further diagnostic testing be limited to three groups: patients for whom the initial evaluation (history, examination, and ECG) suggests an arrhythmic cause; patients who remain anxious to have an explanation for their symptoms; and patients at high risk of ventricular arrhythmias.

This is an observational study and not a clinical trial to determine whether this intervention results in better or cheaper delivery of care than conventional models. Furthermore, there may be other ways of assessing such patients, distinct from standard cardiology outpatient clinics. A simpler service model may be to provide open access ambulatory (Holter) monitoring to general practice. However, we have shown that the clinical assessment is paramount and the Holter much less valuable. Some general practitioners may feel that they do not have the frequency of exposure to interpret the results of the Holter, so while this service may be cheaper and easier to set up, it would not result in the comprehensive clinical evaluation provided by a RAAC. Another option may be to establish a rapid access atrial fibrillation clinic. This was the most common arrhythmia diagnosed and one of the clinic's key achievements was providing a full clinical assessment with early initiation of anticoagulation. This may be a more focused use of resources but would not cater for all patients with arrhythmia related symptoms.

In our institution the establishment of the RAAC has enabled the provision of a comprehensive "rapid cardiology" service for all the common presentations of cardiac disease, incorporating a chest pain, heart failure, and arrhythmia clinic. We have shown that this innovative approach provides a rapid diagnosis, stratifies risk, and leads to prompt initiation of evidence based treatments for patients presenting with suspected new cardiac arrhythmias.

\section{Authors' affiliations}

J L Martins, K F Fox, D A Wood, Cardiovascular Medicine, National Heart and Lung Division, Charing Cross Campus, Imperial College, London, UK

D C Lefroy, Department of Cardiology, National Heart and Lung Division, Hammersmith Campus, Imperial College, London, UK

N S Peters, Department of Cardiology, National Heart and Lung Division, St Mary's Campus, London, UK

T J Collier, Medical Statistics Unit, Department of Epidemiology \& Population Health, London School of Hygiene \& Tropical Medicine, London, UK

\section{REFERENCES}

1 Department of Health. The national service framework for coronary heart disease. London: Stationery Office, 2000.

2 Sutcliffe SJ, Fox KF, Wood DA. How to set up and run a rapid access chest pain clinic. Br J Cardiol 2001;7:692-702.

3 Fox KF, Cowie MR, Wood DA, et al. A rapid access heart failure clinic provides a prompt diagnosis and appropriate management of new heart failure presenting in the community. Eur $J$ Heart Fail 2000;2:423-9.

4 Krahn AD, Manfreda J, Tate RB, et al. The natural history of atrial fibrillation: incidence, risk factors, and prognosis in the Manitoba follow-up study. Am J Med 1995;98:476-84.

5 Benjamin EJ, Wolf PA, D'Agostino RB, et al. Impact of atrial fibrillation on the risk of death: the Framingham heart study. Circulation 1998;98:946-52.

6 Wolf PA, Abbott RD, Kannel WB. Atrial fibrillation as an independent risk factor for stroke: the Framingham study. Stroke 1991;22:983-8.

7 Anon. The effect of low-dose warfarin on the risk of stroke in patients with nonrheumatic atrial fibrillation. The Boston area anticoagulation trial for atrial fibrillation investigators. N Engl J Med 1990;323:1505-11.

8 Petersen P, Boysen G, Godffredsen J, et al. Placebo-controlled, randomised trial of warfarin and aspirin for prevention of thromboembolic complications in chronic atrial fibrillation. The Copenhagen AFASAK study. Lancet 1989;i:175-9.

9 Anon. Stroke prevention in atrial fibrillation study: final results. Circulation 1991;84:527-39 
10 Connolly SJ, Laupacis A, Gent M et al. Canadian atrial fibrillation anticoagulation (CAFA) study. J Am Coll Cardiol 1991;18:349-55.

11 Ezekowitz MD, Bridgers SL, James KE, et al. Warfarin in the prevention of stroke associated with nonrheumatic atrial fibrillation. Veterans affairs stroke prevention in nonrheumatic atrial fibrillation investigators. N Engl J Med 1992;327:1406-12.

12 EAFT (European atrial fibrillation trial) Study Group. Secondary prevention in non-rheumatic atrial fibrillation after transient ischaemic attack or minor stroke. Lancet 1993;342:1255-62.

13 Lip GY, Golding DJ, Nazir M, et al. A survey of atrial fibrillation in general practice: the West Birmingham atrial fibrillation project. Br J Gen Pract 1997;47:285-9.

14 Stafford RS, Singer DE. Recent national patterns of warfarin use in atrial fibrillation. Circulation 1998;97:1231-3.

15 Sudlow M, Thomson R, Thwaites B, et al. Prevalence of atrial fibrillation and eligibility for anticoagulants in the community. Lancet 1998;352:1 167-71.

16 Wheeldon NM, Tayler DI, Anagnostou E, et al. Screening for atrial fibrillation in primary care. Heart 1998;79:50-5.
17 Majeed A Moser K, Carroll K. Trends in the prevalence and management of atrial fibrillation in general practice in England and Wales, 1994-1998: analysis of data from the general practice research database. Heart $2001 ; 86: 284-8$

18 Levy S, Breithardt G, Campbell RW, et al. Atrial fibrillation: current knowledge and recommendations for management. Working group on arrhythmias of the European Society of Cardiology. Eur Heart J 1998; 19:1294-320.

19 Wolf PA, Kannel WB, McGee DL, et al. Duration of atrial fibrillation and imminence of stroke: the Framingham study. Stroke 1983;14:664-7.

20 Frost L, Engholm G, Johnsen S, et al. Incident stroke after discharge from the hospital with a diagnosis of atrial fibrillation. Am J Med 2000; 108:36-40.

21 Underwood MJ, Firmin RK, Jehu D. Aspects of psychological and social morbidity in patients awaiting coronary artery bypass grafting. Br Heart $J$ 1993;69:382-4

22 Zimetbaum P, Josephson ME. Evaluation of patients with palpitations N Engl J Med 1998;338:1369-73.

\section{IMAGES IN CARDIOLOGY}

\section{Cardiac tumour in a neonate with left ventricular oufflow tract obstruction}

D uring routine neonatal examination of a healthy looking female infant on day 1 of life, a grade $2 / 6$ ejection systolic murmur was audible at the left sternal border. Chest $x$ ray and ECG were normal. An echocardiogram revealed a mass $8 \mathrm{~mm}$ in diameter in the left ventricular outflow tract (LVOT), with a pressure gradient of $25 \mathrm{~mm} \mathrm{Hg}$ between the left ventricle and aorta by Doppler study. The mass was seen arising from the interventricular septum just below the aortic valve and there were two small masses at the right ventricular apex (panels A and B ; Ao, aorta; LA, left atrium; LV, left ventricle; RA, right atrium; RV, right ventricle; $\mathrm{T}$, tumour mass). In view of the serious risk for embolic events or total obstruction and sudden death, the tumour mass was resected on the fifth day of life through a transaortic approach (right panel) under cardiopulmonary bypass and cardioplegic arrest. The postoperative period was uneventful and a repeat echocardiogram revealed no residual mass or aortic valve dysfunction. The LVOT was widely patent both anatomically and on Doppler study. Histopathological examination of the mass measuring $9 \times 8 \times$ $7 \mathrm{~mm}$ revealed it to be a rhabdomyoma. Examination of the fundus and ultrasonography of the head and abdomen of the neonate were normal. Since the majority of infants presenting with a cardiac rhabdomyoma develop tuberous sclerosis in later life, prolonged follow up is indicated.

Rhabdomyoma is the most common benign primary cardiac tumour in children and it may be single or multiple. Large tumours may present with arrhythmia, dysfunction of cardiac valves, pericardial effusion, non-immune hydrops

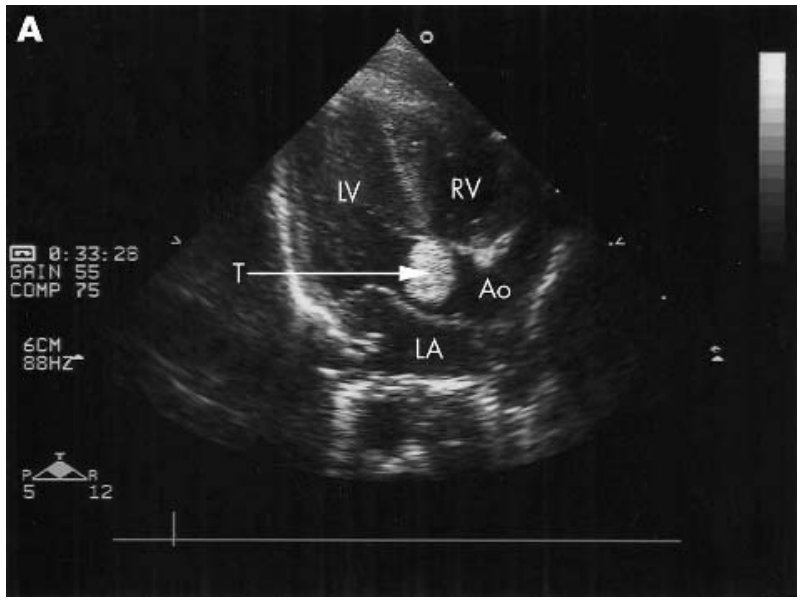

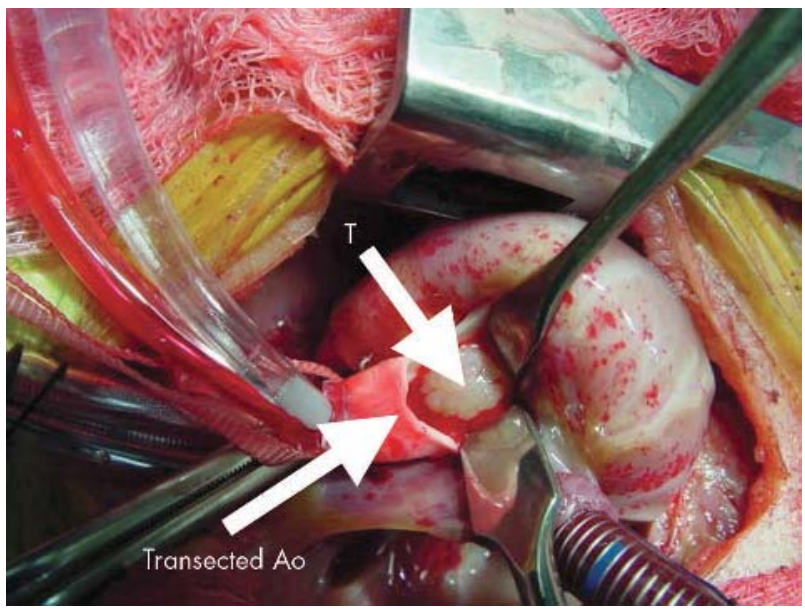

fetalis, congestive heart failure, embolisation, and inflow/ outflow obstruction. Tumour regression mostly occurs in the first two years of life. Surgery is recommended in the presence of obstruction or life threatening arrhythmias.
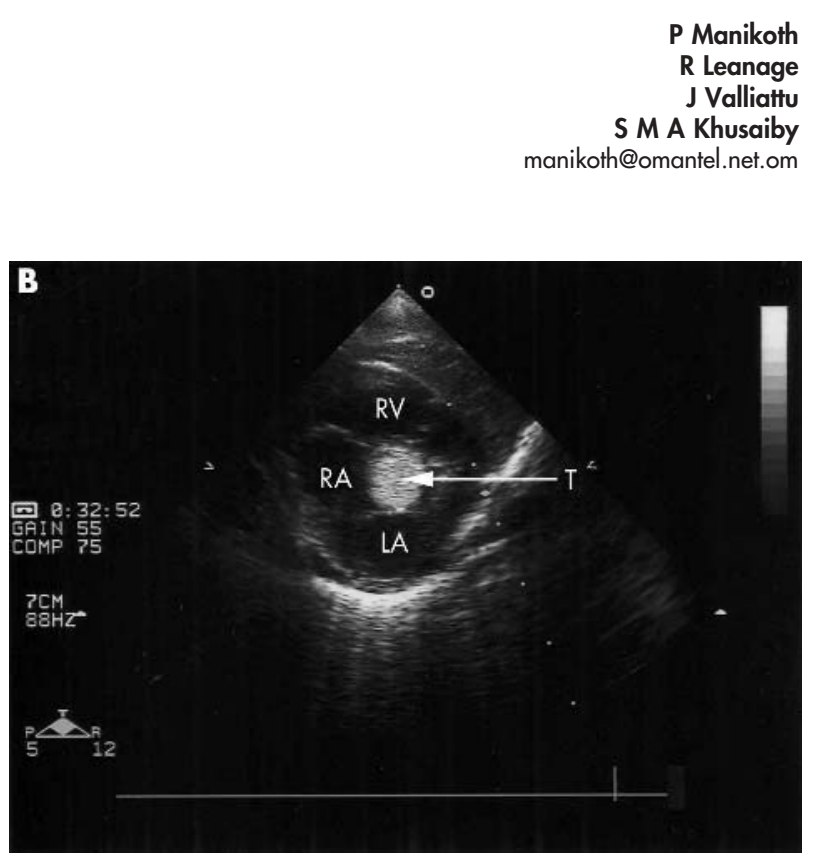